\title{
Surfactante pulmonar exógeno en adultos con síndrome de dificultad respiratoria aguda
}

\author{
Yinet Barrese-Pérez, * ${ }^{*}$ Angela Olga Hidalgo-Sánchez, ${ }^{*}$ Yisel Ávila-Albuerne, * Rolando Uranga-Piña,* \\ Elaine Díaz-Casañas, ${ }^{\S}$ Octavio Fernández-Limia ${ }^{\S}$
}

\author{
*Centro Nacional Coordinador de Ensayos Clínicos (CENCEC), La Habana, Cuba; \\ ${ }^{\ddagger}$ Ministerio de Salud Pública (MINSAP), La Habana, Cuba; ${ }^{\S}$ Centro Nacional de Sanidad Agropecuaria (CENSA), \\ San José de las Lajas, Mayabeque, Cuba. \\ Trabajo recibido: 09-III-2015; aceptado: 08-VII-2015
}

\begin{abstract}
RESUMEN. Objetivo: Determinar el efecto del surfactante pulmonar (Surfacen ${ }^{\circledR}$ ) en la recuperación de la oxigenación en adultos con síndrome de dificultad respiratoria aguda (SDRA). Material y métodos: Adultos entre 18 y 75 años con SDRA, se incluyeron (48 pacientes) en un ensayo clínico fase II, controlado, aleatorizado y multicéntrico. Un grupo (24) recibió tratamiento de surfactante pulmonar cada ocho horas, durante tres días, en dosis de $100 \mathrm{mg}$ totales, junto al tratamiento estándar (oxigenación y ventilación mecánica). El grupo control (24) sólo recibió el tratamiento estándar. El efecto se evaluó a través del índice $\mathrm{PaO}_{2} / \mathrm{FiO}_{2}$, la distensibilidad estática (Cs) y la radiografía de tórax. Otras variables estudiadas fueron la mortalidad, días de intubación, de ventilación mecánica (VM) y de estancia en la Unidad de Cuidados Intensivos (UCI). Resultados: Existieron diferencias estadísticamente significativas $(p=0.0399)$ entre los grupos en el índice $\mathrm{PaO}_{2} / \mathrm{FiO} \mathrm{F}_{2}$, la Cs $(p=0.005)$. No hubo diferencias estadísticas en la radiografía de tórax $(p=0.7177)$, la mortalidad (0.3652), días de intubación endotraqueal $(p=0.2659)$, de VM $(p=0.3582)$ y estancia en la UCI $(p=0.2941)$. Conclusiones: El uso del surfactante pulmonar (Surfacen $\left.{ }^{\circledR}\right)$ junto al tratamiento estándar en adultos con SDRA mejoró significativamente la oxigenación.
\end{abstract}

Palabras clave: Surfactante exógeno, síndrome de dificultad respiratoria aguda, adultos.

ABSTRACT. Objective: To determine the effect of the lung surfactant (Surfacen ${ }^{\circledR}$ ) in the recovery of the oxygenation in adults with acute respiratory distress syndrome (ARDS). Material and methods: Adults between 18 and 75 years with acute respiratory distress syndrome, were included (48 patients) in a clinical trial phase II, controlled, randomized and multicentric. A group (24) received treatment of lung surfactant every eight hours, during three days, in dose of 100 total $\mathrm{mg}$, next to the standard treatment (oxygenation and ventilation mechanics). The group control (24) alone received the standard treatment. The effect was evaluated through the index $\mathrm{PaO}_{2} / \mathrm{FiO}_{2}$, compliance $(\mathrm{Cs})$ and the thorax X-Ray. Other studied variables were the mortality, days of intubation, of ventilation mechanics (VM) and of stay in the Unit of Intensive Cares (UCl). Results: It existed differences statistically significant $(p=0.0399)$ among the groups in the index $\mathrm{PaO}_{2} / \mathrm{FiO}_{2}, \mathrm{compliance}(\mathrm{p}=$ 0.005). There were not statistical differences in the thorax X-Ray $(p=0.7177)$, the mortality $(p=0.3652)$, days of intubation endotracheal $(p=$ $0.2659)$, of ventilation mechanics $(p=0.3582)$ and stay in the UCI $(p=0.2941)$. Conclusions: The use of the lung surfactant (Surfacen $\left.{ }^{\circledR}\right)$ next to the standard treatment in adults with ARDS improved oxygenation significantly.

Key words: Exogenous surfactant, acute respiratory distress syndrome, adults.

\section{INTRODUCCIÓN}

El síndrome de dificultad respiratoria aguda (SDRA) es una insuficiencia respiratoria grave debida a edema no hemodinámico causado por aumento de la permeabilidad de la barrera alvéolo-capilar y secundario a daño pulmonar agudo, siendo un problema frecuente en las Unidades de Cuidados Intensivos (UCI). Descrito en 1967 por Ashbaugh et al. ${ }^{1}$ con una etiología y fisiopatología heterogéneas. El diagnóstico del SDRA está basado en una combinación de criterios clínicos, hemodinámicos y de oxigenación. A partir de las definiciones del Consenso de la Conferencia Americana-Europea (AECC) en $1994^{2}$ se establecieron los criterios diagnósticos que fueron utilizados hasta que en junio de 2012 se publican las conclusiones de una reunión llevada a cabo en Berlín ${ }^{3}$ por encargo de la American Thoracic Society, la Society of Critical Care Medicine, ambas de los Estados Unidos de América, en conjunto con la European Society of Critical Care Medicine. El propósito era alcanzar un acuerdo sobre una nueva definición del SDRA que homogeneizara criterios y que permitiera un fácil reconocimiento de la entidad, ${ }^{4}$ 
así como una mejor y más racional inclusión prospectiva de pacientes en ensayos clínicos.

Estudios recientes sitúan la incidencia del SDRA entre el 4.6 y $8.1 \%$ de los pacientes ingresados en unidades de cuidados críticos, entre el 6.3 y $12.0 \%$ de los pacientes que requieren ventilación mecánica (VM) y alrededor de tres millones de personas/año cuando se refieren a la población general. ${ }^{5-7}$ En Cuba, según el criterio de los especialistas, alrededor del $40 \%$ de los pacientes ingresados en las UCl se les diagnostica SDRA.

Las causas del SDRA son múltiples, pero se reconocen con mayor frecuencia: por afección pulmonar directa y por afección pulmonar indirecta, incluyéndose en esta última el síndrome séptico, los traumatismos no torácicos, las transfusiones múltiples y el secundario a cortocircuito cardiopulmonar. ${ }^{8,9}$

Las tasas de mortalidad por SDRA varían desde un 30 a 75\%, y la mayoría de las muertes son consecuencia de un fallo multiorgánico. ${ }^{2,6} \mathrm{La} V \mathrm{VM}$ con presión positiva al final de la espiración (PEEP) forma parte de la estrategia fundamental del tratamiento en pacientes con fallo respiratorio hipoxémico. ${ }^{8}$

Los surfactantes exógenos se han utilizado como alternativa en el tratamiento del SDRA en numerosos ensayos clínicos, reportándose en todos un beneficio en la función pulmonar medido a través de la mejoría de la oxigenación, sin reducción significativa de la mortalidad, ni para el tiempo de ventilación, ni la estadía en la UCl. ${ }^{10}$

En Cuba se desarrolló y se comercializa un surfactante pulmonar natural $\left(\right.$ Surfacen $\left.^{\circledR}\right)$ que es obtenido a partir del lavado broncoalveolar de pulmones de cerdo a través de un proceso de purificación de los principios activos del que resulta una composición de fosfolípidos (95\%); fundamentalmente dipalmitoilfosfatidilcolina (DPPC), proteínas hidrofóbicas (SP-B y SP-C) 1.5\% y otros lípidos (3.5\%). Es un liofilizado blanco, estéril, conteniendo $50 \mathrm{mg}$ en cada vial. ${ }^{11}$

El objetivo de este estudio fue determinar el efecto del surfactante pulmonar (Surfacen ${ }^{\circledR}$ ) en la recuperación de la oxigenación en adultos con síndrome de dificultad respiratoria aguda.

\section{MATERIAL Y MÉTODOS}

Se diseñó un ensayo clínico fase II, multicéntrico, controlado, aleatorizado y abierto con dos grupos de tratamiento. Los comités de ética de investigación humana de las instituciones hospitalarias participantes (17) aprobaron el protocolo de investigación.

Se evaluaron 254 pacientes, de ellos se incluyó a 48 adultos de 18 a 75 años a quienes se les diagnosticó SDRA según los criterios de Consenso de la Conferen- cia Americana-Europea (AECC) en $1994 .^{2}$ Se obtuvo el consentimiento informado de los familiares de cada paciente incluido. Los criterios de inclusión y exclusión fueron:

\section{Criterios de inclusión}

1. Presencia de SDRA en las primeras 24 horas de diagnosticado, dado por los criterios diagnósticos antes mencionados.

2. PEEP $>5 \mathrm{~cm} \mathrm{H}_{2} \mathrm{O}$.

3. Pacientes cuyos familiares den su consentimiento por escrito de participación en el estudio.

4. Edad comprendida entre 18 y 75 años, ambas inclusive.

Criterios de exclusión

1. Mujeres embarazadas, en período de lactancia o puerperio.

2. Enfermedad pulmonar obstructiva crónica (EPOC).

3. Hipersensibilidad al Surfacen ${ }^{\circledR}$ u otro componente de la formulación.

A los pacientes (24) del grupo A se les practicó tratamiento estándar (oxigenación y ventilación mecánica) y se les administró Surfacen ${ }^{\circledR}$ a través del tubo endotraqueal, cada ocho horas, durante tres días hasta completar nueve aplicaciones, en dosis de $100 \mathrm{mg}$ totales. A los pacientes (24) del grupo B se les practicó tratamiento estándar. En ambos grupos se aplicaron los tratamientos concomitantes requeridos para tratar las enfermedades que originaron el SDRA.

El efecto del tratamiento se evaluó a la hora, a las cuatro y a las ocho horas de cada administración de Surfacen ${ }^{\circledR}$ para el grupo A y en el grupo B en esos mismos tiempos a partir de la evaluación inicial, mediante valoración clínica, gasometría arterial y los parámetros de VM. La variable principal fue el índice $\mathrm{PaO}_{2} / \mathrm{FiO}_{2}$. En la evaluación final se otorgaron las categorías de paciente respondedor cuando el índice $\mathrm{PaO}_{2} / \mathrm{FiO}_{2}$ alcanzó valores mayores a $200 \mathrm{mmHg}$ y evolucionó satisfactoriamente con recuperación del SDRA y paciente no respondedor cuando este índice no alcanzó valores mayores a $200 \mathrm{mmHg}$ y no evolucionó satisfactoriamente a la recuperación del SDRA.

Las variables secundarias fueron la distensibilidad estática (Cs), evolución radiológica, la mortalidad, días de intubación, VM y de estancia en la Unidad de Cuidados Intensivos (UCI).

Se realizaron radiografías de tórax diariamente durante los tres primeros días de tratamiento y al 
quinto día, valorando la presencia o ausencia de infiltrados inflamatorios en los campos pulmonares. Cada día se clasificó la evolución radiológica en mejoría, estabilidad o empeoramiento según la disminución, mantenimiento o aumento de los infiltrados pulmonares.

Se controlaron las variables de edad, sexo, color de la piel y clasificación de la causa del SDRA (directas e indirectas).

El proceso de aleatorización se realizó a través de sobres sellados, previamente identificados con un código y precisando el tratamiento para cada paciente de acuerdo con una lista aleatoria generada automáticamente en un ordenador y utilizando el sistema Statistica, de manera que el número total de pacientes del grupo A coincidió con los del grupo $B$.

Para el análisis estadístico se aplicaron los softwares SAS para Windows, versión 9.1.3, SPLUS 6.2 e East 4.0. Se realizaron tests de normalidad para verificar los requisitos de las pruebas t y se hicieron análisis de correlación entre las diversas variables cuantitativas. Además, se emplearon estadígrafos y herramientas descriptivas para ilustrar los resultados, se utilizaron los tests clásicos asociados a tablas de contingencia, como test chi-cuadrada y test exacto de Fisher y se realizaron pruebas de comparación de medias independientes (test T); en casos necesarios, se acudió al test no paramétrico asociado de Mann-Whitney y Wilcoxon. Se consideró diferencia estadísticamente significativa una $p<0.05$.

\section{RESULTADOS}

La muestra se distribuyó homogéneamente entre los grupos en relación con las características iniciales y demográficas, como se puede apreciar en la tabla 1. El promedio de edad fue de 46 años.

El 25.0\% (12 pacientes) interrumpió definitivamente el tratamiento antes de concluir el esquema indicado, no observándose diferencias estadísticamente significativas entre los grupos ( $p=0.1824) ; 6$ pacientes (3 de cada grupo) interrumpieron debido a causas no previstas en el protocolo de investigación, dadas por dificultades presentadas con la disponibilidad de reactivos y equipos para las evaluaciones gasométricas de los mismos (tabla 1).

En la tabla 1 se muestra además el comportamiento del tipo de lesión pulmonar, donde no se encontró diferencias estadísticamente significativas.

En el análisis de efecto teniendo en cuenta la evaluación final dada por las categorías de paciente respondedor y no respondedor (tabla 2), se obtuvo significación estadística a favor del grupo A, tanto en el análisis por intención de tratar; (reducción del riesgo absoluto/RRA de $29.16 \%$ (IC 95\%) y número necesario de pacientes a tratar/NNT de 3.4) como en el análisis por protocolo (RRA de $28.45 \%$ (IC 95\%) y NNT de 3.5 ).

En ambos grupos el índice $\mathrm{PaO}_{2} / \mathrm{FiO}_{2}$ fue mayor o igual a $200 \mathrm{mmHg}$ en la medida que avanzó el tratamiento. Sin embargo, se evidenció que en el grupo A estos valores fueron mayores e incluso, en determi-

Tabla 1. Características de los pacientes.

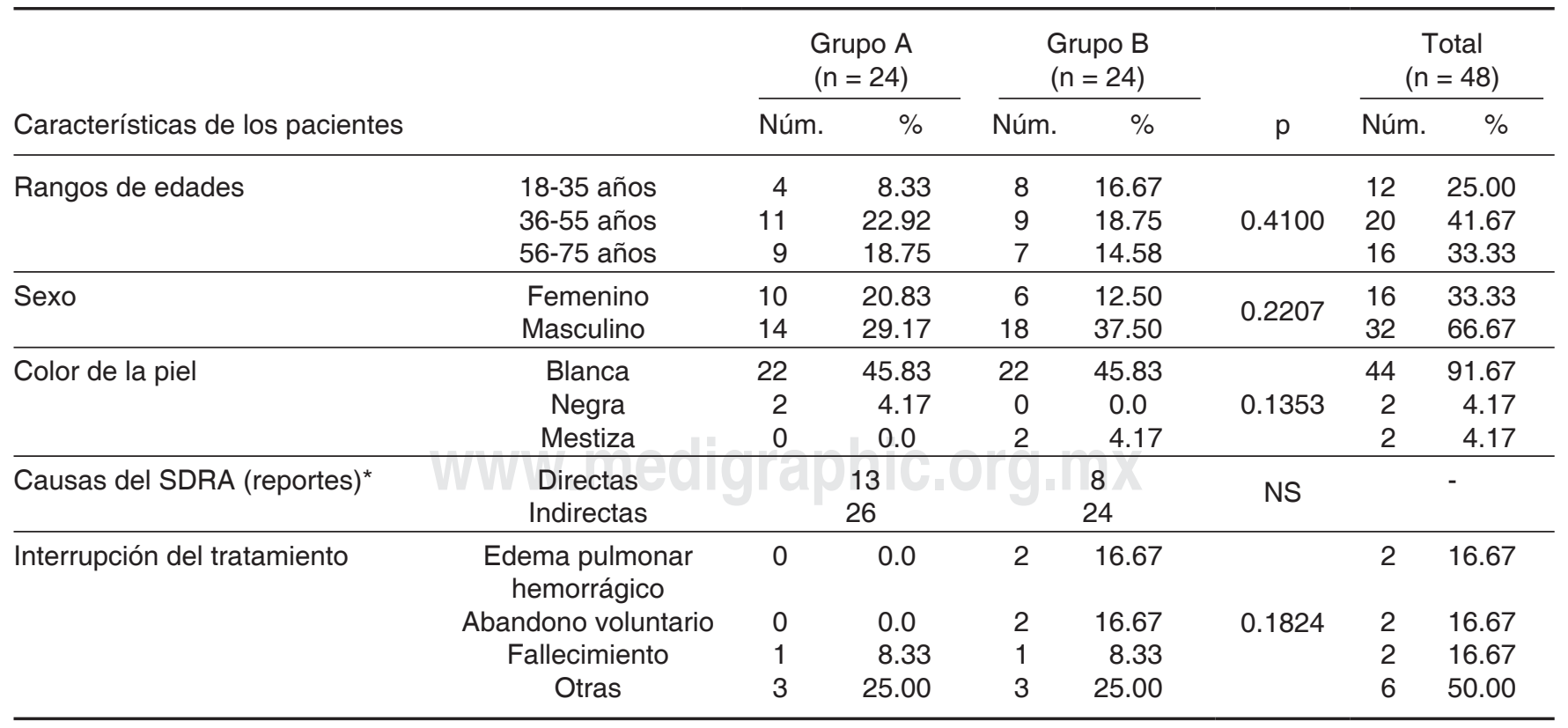

*Se tuvieron pacientes en que se reportó más de una causa de SDRA. 
Tabla 2. Distribución de pacientes según respuesta final.

\begin{tabular}{|c|c|c|c|c|}
\hline Respuesta final & $\begin{array}{c}\text { Grupo A } \\
n(\%)\end{array}$ & $\begin{array}{c}\text { Grupo B } \\
\text { n (\%) }\end{array}$ & $\begin{array}{l}\text { Total } \\
\text { n (\%) }\end{array}$ & $\mathrm{p}$ \\
\hline \multicolumn{5}{|c|}{ Análisis por intención de tratar•• } \\
\hline Respondedor & $17(70.83) \bullet$ & $10(41.67) \bullet$ & $27(56.25)$ & \multirow{3}{*}{0.0399} \\
\hline No respondedor & 7 (29.17)• & $14(58.33) \bullet$ & $21(43.75)$ & \\
\hline Total & $24(50.00)$ & $24(50.00)$ & $48(100.00)$ & \\
\hline \multicolumn{5}{|c|}{ Análisis por protocolo } \\
\hline Respondedor & $17(73.91) \bullet$ & $10(45.45) \bullet$ & $27(60.00)$ & \multirow{3}{*}{0.049} \\
\hline No respondedor & $6(26.09) \bullet$ & $12(54.55)^{\bullet}$ & $18(40.00)$ & \\
\hline Total & $23(51.11)$ & $22(48.89)$ & $45(100.00)$ & \\
\hline
\end{tabular}

- \% respecto a los pacientes analizados por grupo.

- Se otorgó la peor respuesta a 2 pacientes que abandonaron el estudio al momento de la inclusión y 1 al que se le interrumpió erróneamente el tratamiento.

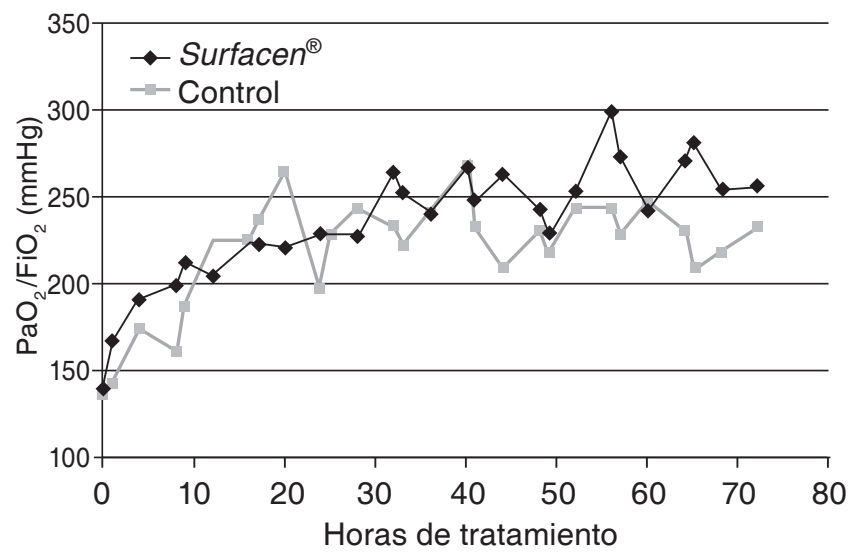

No se evidenció diferencia estadísticamente significativa.

Figura 1. Comportamiento del índice $\mathrm{PaO}_{2} / \mathrm{FiO}_{2}$ según el momento de evaluación.

nadas evaluaciones su superioridad fue significativa (figura 1). Por otra parte, el número de veces en que el índice $\mathrm{PaO}_{2} / \mathrm{FiO}_{2}$ alcanzó valores superiores o iguales a $200 \mathrm{mmHg}$ fue estadísticamente significativo $(\mathrm{p}=$ 0.0170 ) en el grupo $A$, que tuvo una media de 12 ocasiones, respecto al grupo $\mathrm{B}$, en que la media fue de ocho momentos.

El análisis de las variables gasométricas y ventilatorias se muestra en la tabla 3 , evidenciándose un comportamiento estadísticamente significativo posterior a tratamiento en las variables $\mathrm{FiO}_{2}$, PEEP y Cs.

Los resultados de la evaluación radiográfica se pueden apreciar en la tabla 4 en cuya respuesta no se encontró diferencias estadísticamente significativas $(p=0.7177)$.

Los días de intubación endotraqueal, VM y estancia en la $\mathrm{UCl}$ no dependieron del grupo de tratamiento al que se aleatorizaron los pacientes, como se muestra en la tabla 5.

En el estudio falleció el $50.0 \%$ de los pacientes con un comportamiento similar en ambos grupos.

\section{DISCUSIÓN}

Las características de los pacientes incluidos, son similares a las descritas en otros trabajos, sin encontrar diferencias estadísticamente significativas en cuanto al sexo y el color de la piel, aunque predomina el masculino blanco. ${ }^{7,12,13}$

En cuanto a las causas que provocaron el SDRA en este trabajo no hubo diferencias entre los grupos y fueron las indirectas las más frecuentes. En los ensayos realizados por los grupos de Spragg ${ }^{14}$ y Willson ${ }^{15}$ se puso de manifiesto que cuando la causa del SDRA es sistémica se reduce el impacto y la efectividad de la terapia con surfactante exógeno. No obstante se demostró que Surfacen ${ }^{\circledR}$ en las dosis y esquema de tratamiento empleado, fue eficaz en elevar la oxigenación, respuesta que se verifica a través de la relación $\mathrm{PaO}_{2} /$ $\mathrm{FiO}_{2}$, que en el grupo A alcanzó valores superiores a $200 \mathrm{mmHg}$ en más de una ocasión con una frecuencia significativa $(p=0.0170)$ respecto a los reportes del grupo $\mathrm{B}$, así como una reducción del riesgo absoluto a permanecer por debajo de 200 de índice $\mathrm{PaO}_{2} / \mathrm{FiO}_{2}$ de $28.45 \%$; lo que está en correspondencia con otros estudios con similar número de pacientes y diferentes surfactantes exógenos. ${ }^{14,16-18}$ La mejora en la oxigenación incrementa la eficiencia de la ventilación al permitir que disminuyan los valores requeridos de $\mathrm{FiO}_{2}$ y PEEP, así como que aumente la distensibilidad estática, lo que se corrobora en los pacientes del grupo A.

Aunque el número de dosis de Surfacen ${ }^{\circledR}$ no repercutió en la respuesta $(p=0.0930)$, se conoce que dicho surfactante se metaboliza rápidamente y puede inactivarse con facilidad, por lo que el uso de múltiples dosis como las aplicadas en este estudio se relaciona con una disminución de las complicaciones debidas al SDRA y con la tendencia a la mejora clínica de los pacientes. ${ }^{19}$ 
Tabla 3. Comparación de la evolución de la oxigenación entre los grupos.

\begin{tabular}{|c|c|c|c|c|c|c|}
\hline \multirow[b]{2}{*}{ Variables } & \multicolumn{3}{|c|}{ Evaluación inicial } & \multicolumn{3}{|c|}{ Evaluación posterior al tratamiento } \\
\hline & $\begin{array}{c}\text { Grupo A } \\
n=24\end{array}$ & $\begin{array}{c}\text { Grupo B } \\
n=24\end{array}$ & $\mathrm{p}$ & $\begin{array}{c}\text { Grupo A } \\
n=23\end{array}$ & $\begin{array}{c}\text { Grupo B } \\
n=22\end{array}$ & $\mathrm{p}$ \\
\hline $\mathrm{PaO}_{2}(\mathrm{mmHg})$ & 111.57 & 73.00 & 0.2614 & 125.82 & 136.42 & 0.4599 \\
\hline $\mathrm{FiO}_{2}(\%)$ & 1.7 & 0.9 & 0.1992 & 0.68 & 0.59 & $0.0017^{*}$ \\
\hline $\mathrm{PCO}_{2}(\mathrm{mmHg})$ & 51.53 & 40.47 & 0.4934 & 38.69 & 38.34 & 0.3834 \\
\hline $\mathrm{Cs}\left(\mathrm{mL} / \mathrm{cm} \mathrm{H} \mathrm{H}_{2} \mathrm{O}\right)$ & 45.96 & 40.19 & 0.5119 & 48.27 & 36.66 & $0.0343^{*}$ \\
\hline PEEP & 9.12 & 10.70 & 0.1900 & 10.14 & 11.54 & $0.0371^{*}$ \\
\hline
\end{tabular}

Tabla 4. Evaluación radiográfica por grupo de tratamiento al quinto día.

\begin{tabular}{lcccc}
\hline Grupo & Respuesta & $\begin{array}{c}\text { Mejoría } \\
n(\%)\end{array}$ & $\begin{array}{c}\text { Estabilidad } \\
n(\%)\end{array}$ & $\begin{array}{c}\text { Empeoramiento } \\
n(\%)\end{array}$ \\
\hline A $(p=0.7177)$ & Respondedor & $13(65.00)$ & $4(20.00)$ & $0(0.00)$ \\
Datos faltantes: 4 & No respondedor & $2(10.00)$ & $1(5.88)$ & $0(0.00)$ \\
& Total & $15(75.00)$ & $5(25.00)$ & $0(0.00)$ \\
B $(p=0.9400)$ & Respondedor & $3(23.08)$ & $2(15.38)$ & $2(15.38)$ \\
Datos faltantes: 11 & No respondedor & $2(15.38)$ & $2(15.38)$ & $2(15.38)$ \\
& Total & $3(38.46)$ & $4(30.77)$ & $4(30.77)$ \\
\hline
\end{tabular}

Tabla 5. Indicadores hospitalarios.

\begin{tabular}{|c|c|c|c|c|c|c|c|c|}
\hline & & \multicolumn{2}{|c|}{ Grupo A $(n=24)$} & \multicolumn{2}{|c|}{ Grupo B (n= 24) } & \multirow[b]{2}{*}{$\mathrm{p}$} & \multicolumn{2}{|c|}{ Total $(n=48)$} \\
\hline & & $\mathrm{n}$ & $\%$ & $\mathrm{n}$ & $\%$ & & $\mathrm{~N}$ & $\%$ \\
\hline \multirow[t]{2}{*}{ Mortalidad } & Vivos & 10 & 22.73 & 12 & 27.27 & $\begin{array}{c}0.3652 \\
\text { Datos } \\
\text { faltantes: } 4\end{array}$ & 22 & 50.00 \\
\hline & Fallecidos & 13 & 29.55 & 9 & 20.45 & & 22 & 50.00 \\
\hline \multicolumn{2}{|c|}{ Días de intubación endotraqueal } & \multicolumn{2}{|c|}{13.65} & \multicolumn{2}{|c|}{16.63} & 0.2659 & & \\
\hline \multicolumn{2}{|c|}{ Días de ventilación mecánica } & \multicolumn{2}{|c|}{14.00} & \multicolumn{2}{|c|}{15.72} & 0.3582 & & \\
\hline \multicolumn{2}{|c|}{ Días de estancia en la UCl* } & \multicolumn{2}{|c|}{16.34} & \multicolumn{2}{|c|}{22.33} & 0.2941 & & \\
\hline
\end{tabular}

* Unidad de Cuidados Intensivos.

En los pacientes con SDRA se espera que la respuesta radiológica sea tardía debido a la lenta evolución clínica. En este estudio no se detectó diferencias estadísticamente significativas para esta variable independientemente del grupo de tratamiento; sin embargo Rodríguez et al. ${ }^{20}$ reportaron disminución del proceso inflamatorio en un caso pediátrico, atribuyendo este efecto a las propiedades biofísicas, antinflamatorias y antibacterianas del Surfacen ${ }^{\circledR}$.

La mortalidad (50\%) no se modificó con la administración de surfactante y se corresponde con lo reportado para el SDRA. ${ }^{7,21}$ En este sentido Kesecioglu et al. ${ }^{22,23}$ señalan que el tratamiento con surfactante aumenta la supervivencia, lo que se contradice con un estudio posterior realizado por este mismo autor donde disminuyó. ${ }^{12}$ Spragg et al. ${ }^{14}$ tampoco encontraron que el tratamiento con surfactante modificara la mortalidad. Importante resulta que, a pesar de que el mayor número de pacientes fallecidos pertenecían al grupo A, las causas de muerte no se relacionaron con la administración del surfactante, sino a las enfermedades de base de los pacientes en el momento en que les fue diagnosticado el SDRA, lo que es característico de esta afección donde la mayoría de las muertes son a causa de fallo multiorgánico. ${ }^{12}$

En relación con los indicadores hospitalarios se evidenció un comportamiento similar al de otras investigaciones. ${ }^{24} \mathrm{La}$ reducción alcanzada respecto a los días requeridos de VM e intubación endotraqueal y de estancia en la UCI no fue estadísticamente significativa. 
Se puede concluir que el tratamiento combinado de la administración de Surfacen ${ }^{\circledR}$ en bajas dosis repetidas junto a oxigenación y VM, resultó eficaz en mejorar la oxigenación, sin modificar la mortalidad, ni los indicadores hospitalarios.

\section{Agradecimientos}

Agradecemos a los investigadores de las instituciones hospitalarias los coordinadores de la investigación clínica y coordinadores provinciales de ensayos clínicos su participación y colaboración en el estudio, sin los cuales no se hubiera podido llevar a cabo la investigación.

\section{REFERENCIAS}

1. Ashbaugh DG, Bigelow DB, Petty TL, Levine BE. Acute respiratory distress in adults. Lancet 1967;2(7511):319-323.

2. Bernard GR, Artigas A, Brigham KL, et al. The AmericanEuropean Consensus Conference on ARDS. Definitions, mechanisms, relevant outcomes, and clinical trial coordination. Am J Respir Crit Care Med 1994;149(3 Pt 1):818-824.

3. ARDS Definition Task Force, Ranieri VM, Rubenfeld GD, Thompson BT, et al. Acute respiratory distress syndrome: the Berlin Definition. JAMA 2012;307(23):25262533. doi: 10.1001/jama.2012.5669.

4. Costa EL, Amato MB. The new definition for acute lung injury and acute respiratory distress syndrome: is there room for improvement? Curr Opin Crit Care 2013;19(1):16-23. doi: 10.1097/MCC.0b013e32835c50b1.

5. Gaieski DF, Edwards JM, Kallan MJ, Carr BG. Benchmarking the incidence and mortality of severe sepsis in the United States. Crit Care Med 2013;41(5):1167-1174. doi: 10.1097/CCM.0b013e31827c09f8.

6. Goyal M, Houseman D, Johnson NJ, Christie J, Mikkelsen $\mathrm{ME}$, Gaieski DF. Prevalence of acute lung injury among medical patients in the emergency department. Acad Emerg Med 2012;19(9):E1011-1018. doi: 10.1111/j.15532712.2012.01429.x.

7. Mikkelsen ME, Shah CV, Meyer NJ, et al. The epidemiology of acute respiratory distress syndrome in patients presenting to the emergency department with severe sepsis. Shock 2013;40(5):375-381. doi: 10.1097/ SHK.0b013e3182a64682.

8. Villar J, Blanco J, Kacmarek RM. Acute respiratory distress syndrome definition: do we need a change? Curr Opin Crit Care 2011;17(1):13-17. doi: 10.1097/ MCC.0b013e32834271fb.

9. Saharan S, Lodha R, Kabra SK. Management of acute lung injury/ARDS. Indian J Pediatr 2010;77(11):1296-302. doi: 10.1007/s12098-010-0169-z.

10. Raghavendran K, Willson D, Notter RH. Surfactant therapy for acute lung injury and acute respiratory distress syndrome. Crit Care Clin 2011;27(3):525-559. doi: 10.1016/j.ccc.2011.04.005.

11. Manzanares TD, Díaz CE, Alfonso Lorenzo WC, et al. Centro Nacional de Sanidad Agropecuaria. Surfactan- te pulmonar natural de origen porcino. Patente de la República de Cuba A61K 35/42. 1997 Dic 24.

12. Kesecioglu J, Beale R, Stewart TE, et al. Exogenous natural surfactant for treatment of acute lung injury and the acute respiratory distress syndrome. Am J Respir Crit Care Med 2009;180(10):989-994. doi: 10.1164/ rccm.200812-19550C.

13. Brown LM, Kallet RH, Matthay MA, Dicker RA. The influence of race on the development of acute lung injury in trauma patients. Am J Surg 2011;201(4):486-491. doi: 10.1016/j.amjsurg.2010.02.003.

14. Spragg RG, Lewis JF, Wurst W, et al. Treatment of acute respiratory distress syndrome with recombinant surfactant protein C surfactant. Am J Respir Crit Care Med 2003;167(11):1562-1566.

15. Willson DF, Thomas NJ, Markovitz BP, et al.; Pediatric Acute Lung Injury and Sepsis Investigators. Effect of exogenous surfactant (calfactant) in pediatric acute lung injury: a randomized controlled trial. JAMA 2005;293(4):470-476.

16. Amital A, Shitrit $D$, Raviv $Y$, et al. The use of surfactant in lung transplantation. Transplantation 2008;86(11):15541559. doi: 10.1097/TP.0b013e31818a8418.

17. Taut FJ, Rippin G, Schenk P, et al. A Search for subgroups of patients with ARDS who may benefit from surfactant replacement therapy: a pooled analysis of five studies with recombinant surfactant protein-C surfactant (Venticute). Chest 2008;134(4):724-732. doi: 10.1378/ chest.08-0362.

18. Delfín BCA, Rodríguez ÁA. Administración del surfactante exógeno en el síndrome de distress respiratorio agudo. Rev Cub Med Int Emerg 2005;5(1):1-13.

19. Sánchez RC, Torres TJ. Surfactante pulmonar. Rev Ped Elec (en línea) 2004;1(1):45-50. ISSN 0718-0918

20. Rodríguez MVS, Prince MIA, Loret de Mola BY, Díaz CE. Uso de surfactante exógeno en un caso pediátrico de insuficiencia respiratoria aguda hipoxémica: presentación de un caso. AMC [revista en línea]. 2014;18(1):100109. Fecha de consulta: 22 de febrero, 2015. Disponible en: http://scielo.sld.cu/scielo.php?script=sci arttext\&pid=S1025-02552014000100011\&Ing=es.

21. Villar J, Blanco J, Añón JM, et al.; ALIEN Network. The ALIEN study: Incidence and outcome of acute respiratory distress syndrome in the era of lung protective ventilation. Intensive Care Med 2011;37(12):1932-1941. doi: 10.1007/s00134-011-2380-4.

22. Kesecioglu J, Schultz M, Lundberg D, Lauven P, Lachmann B. Treatment of acute lung injury (ALI/ARDS) with surfactant [abstract]. Am J Respir Crit Care Med 2001;163:A819.

23. Kesecioglu J, Schultz M, Maas J, De Wilde R, Steenken E, Lachmann B. Treatment of acute lung injury and ARDS with surfactant is safe [abstract]. Am J Respir Crit Care Med 2004;169:A349.

24. Willson DF, Thomas NJ, Tamburro R, et al.; Pediatric Acute Lung and Sepsis Investigators Network. Pediatric calfactant in acute respiratory distress syndrome trial. Pediatr Crit Care Med 2013;14(7):657-665. doi: 10.1097/ PCC.0b013e3182917b68. 


\section{$\triangle$ Correspondencia:}

Dra. Yinet Barrese Pérez

Centro Nacional Coordinador de Ensayos Clínicos (CENCEC). Calle 5ta. A e/60 y 62, Playa, La Habana, Cuba.

Correo electrónico:

yinet@cencec.sld.cu; yinetbp@infomed.sld.cu
Conflicto de intereses:

«Declaramos que la Ing. Elaine Díaz Casañas y el Dr. Octavio Fernández Limia tienen conflicto de intereses pues son plantilla del centro promotor, pero no participaron en la obtención, registro y evaluación de los datos, sólo en el diseño y en la discusión de los resultados». 